EPJ Web of Conferences 59, 03010 (2013)

DOI: $10.1051 /$ epjconf/20135903010

(C) Owned by the authors, published by EDP Sciences, 2013

\title{
Fast electron beam guiding for effective core heating
}

\author{
T. Johzaki ${ }^{1,5}$, A. Sunahara ${ }^{1}$, S. Fujioka ${ }^{2}$, H. Nagatomo ${ }^{2}$, H. Sakagami ${ }^{3}$ \\ and K. Mima ${ }^{2,4}$ \\ ${ }^{1}$ Institute for Laser Technology, Osaka, Japan \\ ${ }^{2}$ Institute of Laser Engineering, Osaka University, Suita, Osaka, Japan \\ ${ }^{3}$ National Institute for Fusion Science, Toki, Japan \\ ${ }^{4}$ The Graduate School for the Creation of New Photonics Industries, Hamamatsu, Japan \\ ${ }^{5}$ Graduate School of Engineering, Hiroshima University, Higashi-Hiroshima, Japan
}

\begin{abstract}
In cone-guiding fast ignition, the guiding of fast electron beam with significantly large beam divergence is one of the most important issues for achieving efficient core heating. We proposed two guiding schemes; one is the "Tongari" tip guiding by resistive magnetic fields and the other is the guiding by externally applying axial magnetic fields. The guiding performances for these two schemes are demonstrated by Particle-In-Cell and Fokker-Planck simulations.
\end{abstract}

\section{INTRODUCTION}

In cone-guiding electron-driven fast ignition [1], fast electrons generated inside the cone propagate over the distance of $50 \mu \mathrm{m} \sim 100 \mu \mathrm{m}$ to the imploded dense core, and then heat the core up to ignition temperature. Thus, the fast electron beam control (e.g., enhancement of energy coupling of heating laser to fast electrons, optimization of fast electron energy spectrum, reduction of beam divergence and beam guiding to core) is one of the most crucial issues to achieve the ignition and high gain in fast ignition.

According to the previous work [2], the fast electron beam has large beam divergence (full angle of $\sim 100$ degree). Such a large beam divergence could be one of the main factors for lowering the core heating efficiency. There are some ideas on beam guiding using self-generated magnetic fields, e.g., extended double cone [2] and layered cone with guiding wire [3]. The other idea is the guiding by externally applied magnetic fields along to the beam propagation direction [4].

In the present paper, on the basis of 2D Particle-In-Cell (PIC) and Fokker-Planck (FP) simulations, we evaluate the fundamental performances of beam guiding due to the self-generated resistive magnetic fields and the external magnetic fields, and propose a target design for beam guiding to improve the heating efficiency.

\section{FAST ELECTRON BEAM DIVERGENCE}

The fast electron beam divergence was evaluated by 2D PIC simulations [2], where the Au cone with open angle of 30 degree was irradiated by intense laser with intensity of $3 \times 10^{19} \mathrm{~W} / \mathrm{cm}^{2}$ and duration of $1 \mathrm{ps}$, and the fast electron distribution function $f_{\mathrm{fe}}(E, \theta, y, t)(E, \theta, y$ and $t$ are energy, pitch angle, transverse position and time) were observed at the cone tip. The details of simulation condition and results are shown in Ref. [2]. The "particle" and "energy" angular distributions of fast electron beam are evaluated by $f_{f e, n}(\theta)=\iiint f_{f e}(E, \theta, y, t) d t d y d E$ and $f_{\mathrm{fe}, \mathrm{E}}(\theta)=\iiint E \cdot f_{\mathrm{fe}}(E, \theta, y, t) d t d y d E$. The

This is an Open Access article distributed under the terms of the Creative Commons Attribution License 2.0, which permits unrestricted use, distribution, and reproduction in any medium, provided the original work is properly cited. 

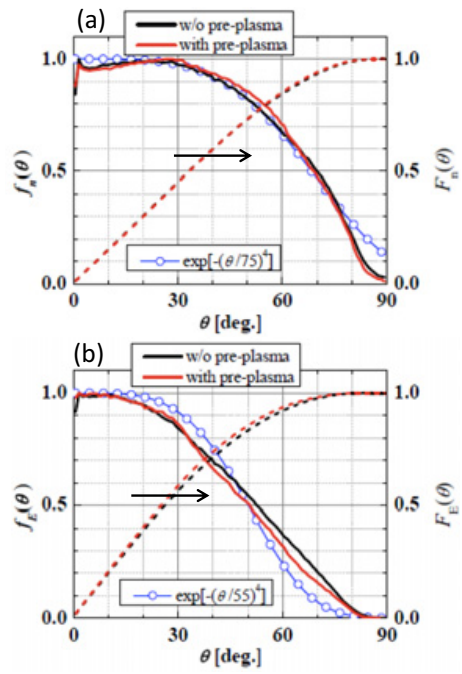

Figure 1. An example of (a) particle and (b) energy angular distributions of fast electron beam evaluated by 2D PIC simulation [2].

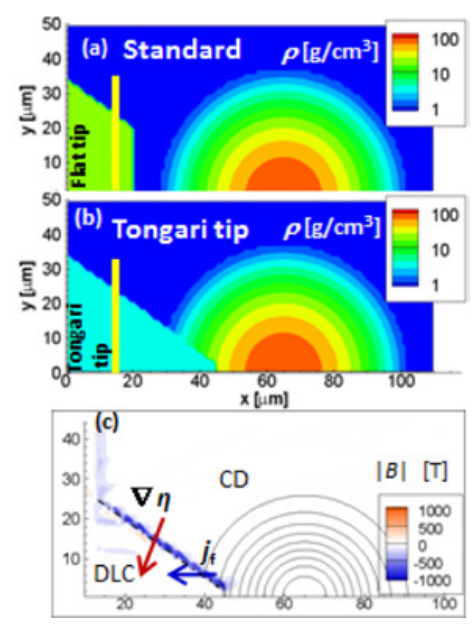

Figure 2. Spatial profiles of (a) inital density of standard Au flat top tip and (b) DLC Tongari tip and (c) resistive magnetic field at $500 \mathrm{fs}$. The yellow lines in (a) and (b) show the beam injection points.

typical angular distributions are shown in Fig. 1, where $F_{\mathrm{fe}, \mathrm{n}}(\theta)=\int_{0}^{\theta} f_{\mathrm{fe}, \mathrm{n}}\left(\theta^{\prime}\right) d \theta^{\prime} / \int_{0}^{2 \pi} f_{\mathrm{fe}, \mathrm{n}}\left(\theta^{\prime}\right) d \theta^{\prime}$ and $F_{\mathrm{fe}, \mathrm{E}}(\theta)=\int_{0}^{\theta} f_{\mathrm{fe}, \mathrm{n}}\left(\theta^{\prime}\right) d \theta^{\prime} / \int_{0}^{2 \pi} f_{\mathrm{fe}, \mathrm{E}}\left(\theta^{\prime}\right) d \theta^{\prime}$ are also plotted. The "particle" and "energy" angular distributions are fitted by exponential functions, $A \exp (-\theta / \Delta \theta)$. The values of $\Delta \theta$ are 75 degree and 55 degree for $f_{\mathrm{fe}, \mathrm{n}}(\theta)$ and $f_{\mathrm{fe}, \mathrm{E}}(\theta)$. The $50 \%$ of beam energy is contained within 30 degree and $90 \%$ of beam energy within 60 degree. Because of such a large beam divergence, most of the fast electrons do not hit the core, which results in the poor heating efficiency. One possibility is the control of laserplasma interactions to reduce the beam divergence, but the control of non-linear process seems to be quite difficult. So we consider the beam guiding. 
Table 1. Summary of guiding performance of Tongari tip.

\begin{tabular}{|l|l|l|l|l|}
\hline $\begin{array}{l}\text { Tongari tip } \\
\text { material }\end{array}$ & $\mathrm{B}_{\max }[\mathrm{T}]$ & $E_{\text {cone }} *$ & $E_{\text {core }} *$ & $\Delta T_{e 0}^{*}$ \\
\hline $\mathrm{Au}$ & 1250 & 1.9 & 0.8 & 1.0 \\
\hline $\mathrm{Cu}$ & 1020 & 1.8 & 1.0 & 1.4 \\
\hline $\mathrm{Al}$ & 977 & 1.3 & 1.3 & 1.8 \\
\hline $\mathrm{DLC}$ & 723 & 1.4 & 1.3 & 1.9 \\
\hline
\end{tabular}

$*$ The values of deposited energies in cone tip $E_{\text {cone }}$ and in core $E_{\text {core }}$, and electron temperature enhancement at core center $\Delta T_{e} 0$ due to heating are normalized by the values for the standard Au flat top cone tip ( $5 \mu \mathrm{m}$ thickness) case.

\section{RESISTIVE GUIDING BY "TONGARI" CONE TIP}

The magnetic field growth in collisional plasmas is derived from Faraday's law and Ohm's law, together with the assumption of quasi neutrality for beam and return currents $\left(\vec{j}_{\text {fe }}=-\vec{j}_{\text {be }}\right)$;

$$
\frac{\partial \vec{B}}{\partial t}=\eta\left(\nabla \times \vec{j}_{f e}\right)+\nabla \eta \times \vec{j}_{f e},
$$

where $\eta$ is the resistivity. Previously, the self-pinch of fast electron beam due to the first term was expected [5, 6]. However, for the case of fast electron beam with large divergence, it does not work well. The second term means the magnetic field generation due to gradient of resistivity, which works to confine the fast electrons in the higher-resistivity region [7]. To guide the fast electron beam to the core, we propose "Tongari" cone tip (Tongari is Japanese word, means "pointed"). If using higher-Z material for the tip compared with the surrounding imploded plasma, the confinement field will be generated at the material contact surface. To confine the fast electrons whose energy is a few MeV, the required strength of magnetic field is a few kT. Though a high- $\mathrm{Z}$ material, such as Au, is preferable for generating the strong magnetic field, the energy loss and scattering of fast electrons become large in the high- $\mathrm{Z}$ material. So, we estimate the guiding performance for different cone materials ( $\mathrm{Au}, \mathrm{Cu}$, Al, DLC) by Fokker-Planck simulations where we used the fast electron beam profiles obtained from 2D PIC simulations [2] for 30 degree cone without pre-plasma and spherically compressed CD core (Gaussian density profile with $200 \mathrm{~g} / \mathrm{cm}^{2}$ density at the core, $\rho R=0.12 \mathrm{~g} / \mathrm{cm}^{2}$ and uniform temperature of $300 \mathrm{eV}$ ) was assumed (Fig. 2(a)-(b)). The spatial profile of magnetic field for the case of DLC Tongari tip at $t=500 \mathrm{fs}$ is shown in Fig. 2(c). Due to the energy deposition by fast electron beam, the DLC tip is rapidly heated, and then the gradient of resistivity at cone-plasma interface becomes smaller. Thus the growth of magnetic field is saturated at this moment. The strength of magnetic field is $\sim 0.7 \mathrm{kT}$, which is not enough to confine the fast electron with the energy of several MeV. But, the relatively lowenergy $(\sim 1 \mathrm{MeV})$ and small-pitch-angle electrons can be confined and guided to the core. The transport simulation results are summarized in Table 1 . The strength of confinement magnetic field generated at the cone-plasma interface is about $1 \mathrm{kT}$ and it increases with material-Z. On the other hand, the collisional effects are also large in the high- $Z$ material, i.e., the energy deposition in the cone is larger for the higher- $\mathrm{Z}$ material. As the results, the core heating rate for the $\mathrm{Au}$ and $\mathrm{Cu}$ Tongari tip cases is smaller than that for the standard Au flat tip. The core heating rate is enhanced for the Al and DLC cone cases, which means that the low-Z material such as Al and DLC is preferable as the Tongari tip.

\section{GUIDING BY EXTERNALLY APPLIED AXIAL MAGNETIC FIELDS}

Under the sufficiently-strong axial magnetic fields, the generated fast electrons twist around the magnetic field lines and are expected to be guided into the core. In addition, the fast electron stopping rage becomes effectively shorter due to the gyro-motion. The core heating is mainly done by the fast electrons of which energy is less than $10 \mathrm{MeV}$. The heating spot size required for efficient ignition is 

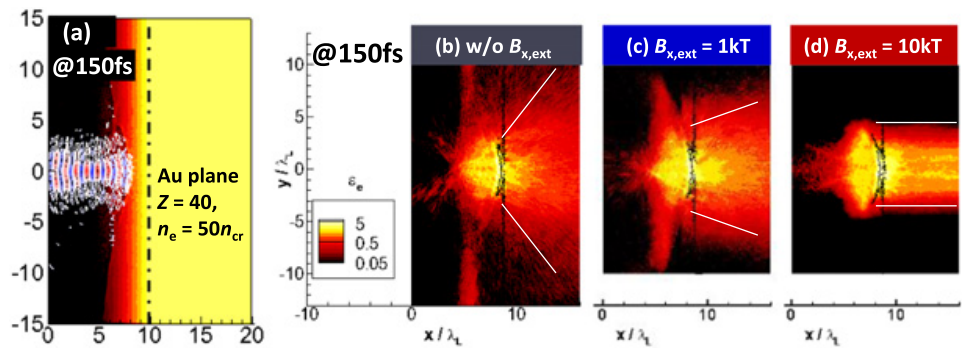

Figure 3. Spatial profiles of (a) electron number density $n_{e}$ normalized by $n_{c r}$ and (b) $\sim$ (d) fast electron energy density observed at $150 \mathrm{fs}$ for $B_{\mathrm{x}, \mathrm{ext}}=0,1,10 \mathrm{kT}$.

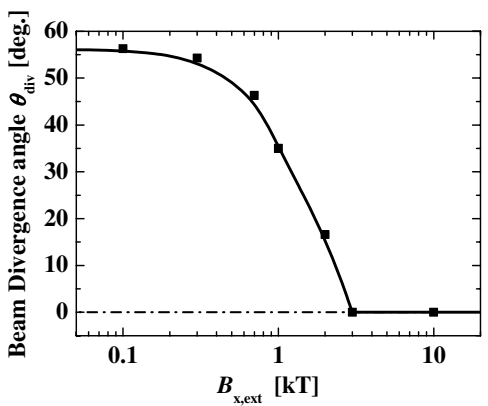

Figure 4. Beam divergence angle $\theta_{\text {div }}$ as a function of $B_{\mathrm{x}, \mathrm{ext}}$.

$\sim 30 \mu \mathrm{m}$ diameter. Thus, the fast electrons of which energies are less than $10 \mathrm{MeV}$ should be confined in a few tens $\mu \mathrm{m}$ region (gyro-radius), which requires magnetic field strength of several kT, e.g., the strength of magnetic field of $3.8 \mathrm{kT}(30 \mathrm{kT})$ is required for $10 \mu \mathrm{m}$ gyro-radius of $1 \mathrm{MeV}(10 \mathrm{MeV})$ electron with 60 degree pitch angle. The preliminary evaluation of guiding performance was done by $2 \mathrm{D}$ PIC simulations (collisionless), where we assumed that an Au plane target $\left(50 n_{\mathrm{cr}}, Z=40\right)$ with $1 \mu \mathrm{m}$ scale pre-plasma is irradiated by an intense laser pulse with $1 \mu \mathrm{m}$ wavelength, $10^{19} \mathrm{~W} / \mathrm{cm}^{2}$ intensity, $200 \mathrm{fs}$ flat top ( $30 \mathrm{fs}$ rising) duration and $5 \mu \mathrm{m}$ spot diameter. The external magnetic fields with strength of $B_{\mathrm{x}, \text { ext }}=0 \sim 10 \mathrm{kT}$ are uniformly applied along to the beam propagation direction. In Fig. 3, (a) the electron density profile and (b) $\sim(\mathrm{d})$ the energy density of fast electron beam for $B_{\mathrm{x}, \mathrm{ext}}=0,1$ and $10 \mathrm{kT}$ observed at $150 \mathrm{fs}$ are shown. It is found that the spatial divergence of electron beam $\theta_{\text {div }}$ is $55^{\circ}$ in the case without external field, and it becomes smaller with increasing the strength of magnetic field.

In Fig. 4, the beam divergence $\theta_{\text {div }}$ is plotted as a function of $B_{\mathrm{x}, \text { ext }}$. The value of $\theta_{\text {div }}$ starts to decrease at $B_{\mathrm{x}, \text { ext }}=0.1 \mathrm{kT}$ and reaches $\theta_{\mathrm{div}}=0^{\circ}$ at $B_{\mathrm{x} \text {,ext }}=3 \mathrm{kT}$. For further strong $B_{\mathrm{x}, \mathrm{ext}}(<3 \mathrm{kT})$, the beam radius becomes smaller due to smaller gyro-radius. These results indicate that the effective guiding can be achieved with $B_{\mathrm{x}, \text { ext }}>$ a few kT. Such a high field could be achieved by implosion [8] of spherical shell target with externally applied magnetic field [9]. Further investigation including magnetic field compression and core heating is indispensable. Especially mirror effect is important since the magnetic field does not compress in the cone region, so the ratio of magnetic field strength between the generation point and compressed dense core region might be $\sim 100$.

\section{CONCLUDING REMARKS}

We demonstrated the guiding performance of two types of guiding concepts (i.e., the resistive guiding by Tongari tip and the externally applied axial magnetic fields). The evaluations are still preliminary ones, 


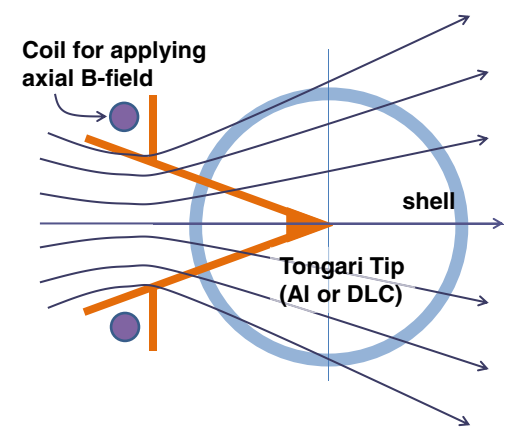

Figure 5. Schematic view of a cone-shell target with two guiding concepts.

so further study is needed. Also, the experimental evidence is indispensable. We propose the guiding scheme by combination of two guiding concepts (Fig. 5) and plan to introduce it into the next FIREX-I integrated experiments.

This work was supported by JSPS, Core-to-Core Program (19003), Photon Pioneers Center in Osaka University. The authors are grateful for the support of the computer room of ILE and the cyber-media center at Osaka University.

\section{References}

[1] R. Kodama, et al., Nature 418, 933 (2002).

[2] T. Johzaki, et al., Nucl. Fusion 51, 073022 (2011).

[3] P. A. Norreys, "Recent Energy Transport Experiments on the Vulcan Petawatt Laser Facility", presented at 37th EPS conf. on Plasma Phys., June 2010, Dublin, Ireland.

[4] D. J. Strozzi, et al., Bull. APS 55, 68 (2010).

[5] R. L. Robinson, and M. Sherlock, Phys. Plasmas 14, 083105 (2007).

[6] T. Johzaki, et al., J. Phys. Conf. Series 112, 022091 (2008).

[7] B. Ramakrsishna, et al., Phys. Rev. Lett. 105, 135001 (2010).

[8] P. Y. Chan, et al., Phys. Rev. Lett. 107, 035006 (2011).

[9] C. Courtois, et al., J. App. Phys. 98, 054913 (2005). 